\title{
An Adaptive wavelet transformation filtering algorithm for improving road anomaly detection and characterization in vehicular technology
}

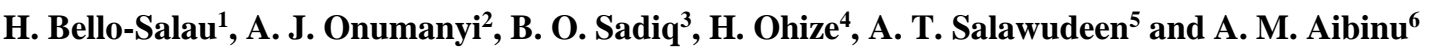 \\ 1,3,5 Department of Computer Engineering, Ahmadu Bello University, Nigeria \\ ${ }^{2}$ Department of Telecommunication Engineering, Federal University of Technology, Nigeria \\ ${ }^{4}$ Department of Electrical and Electronics Engineering, Federal University of Technology, Nigeria \\ ${ }^{6}$ Department of Mechatronics Engineering, Federal University of Technology, Nigeria
}

\begin{abstract}
Article Info
Article history:

Received Feb 20, 2019

Revised Apr 8, 2019

Accepted Apr 15, 2019

\section{Keywords:}

Accelerometer

Road Anomaly

Scale Space Filter

Threshold

Wavelet Transform

ABSTRACT

Accelerometers are widely used in modern vehicular technologies to automatically detect and characterize road anomalies such as potholes and bumps. However, measurements from an accelerometer are usually plagued by high noise levels, which typically increase the false alarm and misdetection rates of an anomaly detection system. To address this problem, we have developed in this paper an adaptive threshold estimation technique to filter accelerometer measurements effectively to improve road anomaly detection and characterization in vehicular technologies. Our algorithm decomposes the output signal of an accelerometer into multiple scales using wavelet transformation (WT). Then, it correlates the wavelet coefficients across adjacent scales and classifies them using a newly proposed adaptive threshold technique. In addition, it uses a spatial filter to smoothen further the correlated coefficients before using these coefficients to detect road anomalies. Our algorithm then characterizes the detected road anomalies using two unique features obtained from the filtered wavelet coefficients in order to differentiate potholes from bumps. The findings from several comparative tests suggest that our algorithm successfully detects and characterizes road anomalies with high levels of accuracy, precision and low false alarm rates as compared to other known methods.
\end{abstract}

Copyright $(0) 2019$ Institute of Advanced Engineering and Science. All rights reserved.

Corresponding Author:

H. Bello-Salau,

Department of Computer Engineering,

Ahmadu Bello University Zaria,

Kaduna State, Nigeria.

Email: bellosalau@abu.edu.ng

\section{INTRODUCTION}

Road anomalies are observable deformities on a typical road surface that deviate from standard expected conditions. They are also considered as obstructions noticed on the surface of asphalt roads that hinder the smooth flow of traffic [1]. These anomalies are usually in the form of rutting, potholes, cracks and speed bumps. Road anomalies are sometimes responsible for road traffic accidents, which often lead to the loss of lives and properties, driving discomfort, increase in vehicular fuel consumption, as well as wear and tear of vehicles [2-4]. Despite continuous efforts to repair anomalous roads and ensuring strong adherence to road construction standards, road anomalies still persist and remain part of the life cycle of most roads. Thus, before road anomalies are noted and corrected by maintenance agencies, it remains pertinent to develop detection and warning systems in vehicular technologies to improve road navigation with the aim to prevent incessant cases of road anomaly induced accidents. 
A popular sensor used in modern vehicular technologies to monitor road surface conditions and to detect road anomalies is the Accelerometer [3, 5-10]. An Accelerometer is a device that measures the acceleration of a body relative to g-force [11]. Though Accelerometers have become widely used in this regard, nevertheless, a major problem with their use is the high noisy content produced in the sensor's output signal [10]. The accelerometer's high noise content stems from its high sensitivity to slight changes in movement or vibration of an object, which is a vehicle in our case. Thus, this high degree of randomness associated with the accelerometer's measurements makes road anomaly detection a difficult process, while making road anomaly characterization an even more challenging task. Thus, an approach in this regard is to develop adaptive threshold estimation techniques suited for classifying the output measure of an accelerometer with the aim to improve road anomaly detection and characterization in vehicular technologies. The development of such a technique motivated the work reported in this paper.

Consequently, in this paper, we have developed an adaptive threshold technique (ATT) used within a Wavelet Transformation Scale Space Filtering (WSSF) algorithm to improve road anomaly detection and characterization in vehicular technologies. The ATT and the WSSF algorithm are combined to constitute the Adaptive Wavelet Transformation Scale Space Filtering (AWSSF) algorithm. The AWSSF processes the accelerometer's measured output by decomposing it into multiple scales and correlating across adjacent scales, while noise samples are filtered using a spatial filter.

The AWSSF's output is then fed to a Road Anomaly Characterization Algorithm (RACA) developed in [12]. Characterization was achieved using two distinct patterns extracted from an accelerometer's output measure to differentiate potholes from bumps. Following our approach, the contribution of this paper entails the development of an AWSSF algorithm that adapts to the dynamic noise level of an accelerometer's output measure to improve road anomaly detection and characterization in vehicular technologies.

The rest of the paper is structured as follows: related work is presented in section 2, an overview of WSSF algorithm and the proposed adaptive threshold technique is presented in section 3 , the proposed AWSSF algorithm is presented in section 4, section 5 presents the results and discussions while, conclusion is drawn in section 6 .

\section{RELATED WORK}

Some approaches for road anomaly detection based on machine learning concepts are noted in $[13,14]$. These approaches though accurate, nevertheless are quite complex owing to the training process required, as well as their dependence on the type/size of the input dataset. Simpler approaches exist such as in [1]. In [1], an accelerometer sensor embedded in a mobile phone along with a gyroscope and inertial sensors were used to monitor road surface conditions. The measured acceleration signals were analyzed using a wavelet transformation technique along with the Support Vector Machine (SVM) algorithm.

These algorithms were trained using statistical parameters such as the standard deviation, the mean, the energy of the signal and the variance. Road anomalies were detected and classified into mild, severe, or span levels. Similarly, a Multilayer Perceptron (MLP) algorithm was proposed in [5] to analyze road anomalies. The experimental results obtained revealed high detection, accuracy and precision rates. A similar approach using a pattern recognition system with an accelerometer sensor and a GPS was proposed in [15] to detect road anomalies. This involved road anomaly classification using the SVM after a pre-processing stage. The experimental results obtained showed that the method in [15] performed better than the method in [5].

A real-time processing algorithm was proposed in [16] to detect potholes using data acquired via a GPS, a video module and an accelerometer. The goal in [16] was to reduce the volume of data transmitted from the sensors to a central processing server. Following [16], other approaches have been reported in $[3,8,10,17-21]$ that considered issues concerning the monitoring, detection, localization and evaluation of road anomalies. We note that these methods lacked the design required for road anomaly characterization into either potholes or speed bumps, which limits the extent of their use.

Consequently, in this paper, we have considered the development of an AWSSF algorithm for the purpose of road anomaly detection and characterization. Our approach ultimately aims to improve road navigation by vehicle drivers to reduce cases of road anomaly induced accidents. Furthermore, our system can be adopted to notify maintenance agencies quickly enough about the presence and whereabouts of these anomalies for quick maintenance response.

\section{RESEARCH METHOD}

In this section, we present the WSSF algorithm as a basis for the adaptive threshold algorithm. We then combine the WSSF and our adaptive threshold algorithm to develop the AWSSF. 


\subsection{Wavelet transformation scale space filtering (SSF) algorithm} expressed as

According to wavelet transformation (WT) theory [22], the typical WT model is generally

$$
f(x)=\underset{j=1}{\stackrel{J}{~}} \stackrel{\bigotimes}{k=1}_{k=1}^{K} \mathrm{Y}_{j, k}(x) W(j, k)
$$

where, $\mathrm{Y}_{j, k}(x)$ is the normalized dual basis function, $J$ is the maximum number of decomposition scales, $K$ is the maximum position in the original signal scale, $W(j, k)$ is the actual scale space decomposition at different resolution scales, $j$ is the index of the scales, and $k$ is the index position in the original signal space. Considering the model in (1), it is important to choose carefully an appropriate basis function $\mathrm{Y}_{j, k}(x)$ in order to compute the wavelet coefficients. We made this choice by visually examining the structure of different known basis functions, such as the Mexican Hat (Morlet), the Haar, Daubechies (db), Coiflet and the Meyer wavelet functions. We then compared these functions to an actual typical signal measured by an Accelerometer. We have presented details concerning this exercise in [10, 12]. Essentially, the comparative exercise in $[10,12]$ was used to identify the wavelet basis function suitable for road anomaly detection and characterization. In this present paper, we adopted the Daubechies-2 (db2) wavelet basis function since it was the most correlated function to most typically measured accelerometer output signals (kindly see [10, 12] for details). We proceed with the WT process by investigating the basis function as follows:

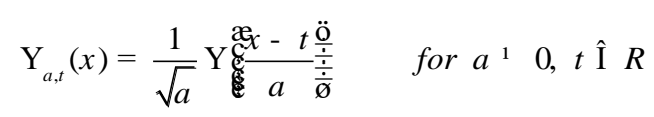

Where, $a$ is the dyadic scale parameter, $1 / \sqrt{a}$ is the resolution, and $t=k a$ is the dyadic translation parameter. The scale variables are discretized, dilated and translated to obtain the dyadic wavelet transforms using

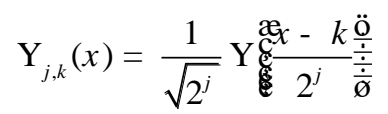

Where $k$ is the translation index $(0, \pm 1, \pm 2, \ldots)$. Thus, (1) - (3) form the basis for the WT theory used in this work. Hence, the WT coefficients $W(j, k)$ corresponding to the sensed signal $f(x)$ were computed using

$$
W(j, k)=\grave{\mathrm{O}}_{-}^{¥} f(x) \mathrm{Y}_{j, k}(x) d x
$$

Where $\mathrm{Y}_{j, k}(x)$ is the set of wavelet function values. We note that if $j$ is increased during the decomposition process, then either the amplitude of the wavelet coefficients remains constant or it increases as the signal edges become positively correlated. Furthermore, we observed that the magnitude of noise samples typically reduces owing to poorer correlation indexes. Consequently, we increased the scale factor in order to isolate noise samples easily from a measured signal set. Thus, this enabled us to identify easily portions in an accelerometer output signal that correspond to potential road anomalies. However, a major challenge lies in effectively adapting the threshold value that classifies the random correlated coefficients. We shall present an adaptive threshold algorithm in Section III-B designed to address this problem. We will then combine it with the Scale Space Filtering (SSF) algorithm described in [22, 23] to develop the AWSSF. Similar to the SSF algorithm, the AWSSF algorithm correlates the wavelet coefficients by directly multiplying wavelet coefficients existing at adjacent scales. The model used in the AWSSF is expressed as

$$
\mathrm{W}_{j}(j, k)=\widetilde{\tilde{O}}_{i=0}^{1} W(j+i, k)
$$


Where $\mathrm{W}$ denotes the spatial correlation function, $J$ is the number of multiplication scales, and $W(j+i, k)$ is the $(j+i) t h$ wavelet coefficients at the $k^{\text {th }}$ scale. By using (5), the AWSSF algorithm identifies noise samples easily and isolates them from features that typically describe road anomalies. This is possible noting that over several scales; signal samples typically produce larger correlation values than noise samples, which produce smaller values. To further explain this process, let the first and second scale wavelet transform coefficients be $W(1, k)$ and $W(2, k)$ respectively. The multiplication of these two scales is expressed as

$$
\mathrm{W}_{2}(1, k)=W(1, k) \cdot W(2, k)
$$

For anomaly detection, $\mathrm{W}_{2}(1, k)$ often produces sharper and more pronounced response values than either $W(1, k)$ or $W(2, k)$. This may be because $\mathrm{W}_{2}(1, k)$ attains larger magnitudes as compared to either $W(1, k)$ or $W(2, k)$. Thus, $\mathrm{W}_{2}(1, k)$ is used in the SSF algorithm instead of $W(1, k)$ or $W(2, k)$. We summarize the steps in the SSF algorithm as follows:

a. $\quad W(j, k)$ is computed using (4) as the set of wavelet coefficients at each scale.

b. At each scale, the power of the correlated data $\mathrm{W}_{2}(j, k)$ is rescaled to match $W(j, k)$.

c. If the new absolute value of $\mathrm{W}_{2}(j, k)$ is greater than or equal to the absolute value of $W(j, k)$ when compared at the scale points $(\mathrm{j}, \mathrm{k})$, then an edge is identified.

d. The edge position and its corresponding $W(j, k)$ value are saved.

e. All identified edges in $\mathrm{W}_{2}(j, k)$ and $W_{(j, k)}$ are then extracted. After extracting the first round of edges at position $(j, k)$, the data points left in $\mathrm{W}_{2}(j, k)$ and $W(j, k)$ are denoted as $\mathrm{W}_{2}(j, k)$ and $W \not(j, k)$.

f. The next edge in the signal is extracted from $W(j, k)$ and $\mathrm{W}_{2}(j, k)$ by rescaling the power of $\mathrm{W}_{2}(j, k)$ to that of $W \varnothing(j, k)$ and then comparing their absolute values.

g. This process of normalizing the power, comparing the data values and extracting edge information is iterated many times until the power of the un-extracted data points in $W(j, k)$ at the scale points $(j, k)$ are approximately equal.

h. The final filtered signal is then stored in $W_{n e w}(j, k)$.

Having established the SSF algorithm, we shall describe next the adaptive threshold technique and then deploy it in Section III-C in the AWSSF algorithm.

\subsection{Adaptive threshold technique}

We describe a new adaptive threshold technique (ATT) suited for use in the SSF algorithm. The ATT adapts automatically to the fluctuating noise content of the correlated wavelet coefficients. The typical SSF algorithm uses a fixed threshold approach that requires careful fine-tuning of several input datasets in order to determine a candidate value. Nevertheless, since the wavelet coefficients are random, the fixed threshold typically fails, thus undermining the algorithm's performance in the end. Consequently, we propose to adapt the SSF's threshold value $T_{t h}$ using

$$
T_{t h}=\frac{K}{N} \underset{i=1}{\stackrel{\circ}{a}} x_{i}
$$

where $N$ is the interval width, $x_{i}$ is the sample acceleration for $i=1,2, \ldots, N$, and $K$ is a constant empirically set to $\mathrm{K}=4$ for improved performance. We obtained this value following several experiments conducted to determine typically maximum values of a set of wavelet coefficients. We shall adapt this adaptive approach in the SSF in the next section. Nevertheless, we compared our approach with other adaptive methods such as the Recursive one-sided hypothesis testing (ROHT) Algorithm [24] and the Otsu multi-thresholding technique [24-26]. Our findings in this regard will be discussed in Section IV. 


\subsection{Adaptive wavelet transforms scale space filtering (AWSSF) algorithm}

Our AWSSF summarized in Algorithm 1 was developed by combining the ATT and the SSF algorithm to classify effectively the correlated wavelet coefficients. The AWSSF passes the classified coefficients through a spatial filter designed based on a unit step rectangular window function (see step 8 of Algorithm 1). It uses a counter to determine the number of anomalies present in the final denoised signal $N S$ obtained in step 11 of Algorithm 1. To characterize the detected road anomalies into potholes or speed bumps, the output of the AWSSF algorithm NS used for detection is fed into the Road Anomaly Characterization Algorithm (RACA) proposed in [12]. RACA is summarized in Algorithm 2. The window size winsig was set at 30 samples in our work to process the detected anomalies NS. We arrived at this value after conducting several experiments. This was used to create a variable NSD that contains zero values up to the length of $N S$. We used a flag $v$ to mark the beginning of each window sample length and initialised $v=1$ along with the sample index counter $W$. RACA also sets the count of the characterized anomalies to 0 in lines 5 and 6. The accelerometer output samples in each window are checked (in Line 7) and windowed samples with values less than 0 and whose immediate signal sample value is greater than or equal to 0 are characterized as speed bumps. Furthermore, any windowed signal sample with an immediate sample values less than or equal to 0 is characterized as a pothole. For further details concerning RACA, readers are kindly referred to [12].

\section{RESULTS AND DISCUSSION}

In this section, the performance of the AWSSF algorithm is evaluated. Road anomalies were characterized using different noisy measured acceleration signals based on distinct features produced by RACA whose output pattern is shown in Figure 1(a) and (b) for potholes and bumps, respectively (reference to [12]). The AWSSF algorithm was applied to several datasets obtained from different road terrains made accessible in $[10,12]$. However, since our work focuses on automating the static threshold value used to detect and characterize road anomalies, thus we evaluated and compared the AWSSF algorithm using different known adaptive threshold techniques, as well as with the optimal static threshold approach used in [12]. Furthermore, we used the following metrics to evaluate the AWSSF: accuracy, precision, and false positive rate, which were computed relative to the dataset considered at the test time. Here, we present in Table 1 and 2 the performance analysis of two representative datasets describing potholes and bumps, respectively.

The results in Table 1 indicate that our ATT automatically sets the scale space threshold to 0.0094 , which provided a $100 \%$ detection rate concerning road anomalies and a false positive rate of less than $0.2 \%$. Other results indicate $100 \%$ accuracy and $75 \%$ precision rate for our ATT. This performance is followed closely by the manual optimal static threshold value of 0.0060 , which achieved a $100 \%$ detection rate, a false alarm rate of $0.37 \%$, accuracy and precision rates of $99.63 \%$ and $60 \%$, respectively. The ROHT algorithm achieved a detection rate of $33.33 \%$, which was better than the Otsu Multi Threshold algorithm with a $0 \%$ detection and precision rate.

Algorithm 1: AWSSF algorithm for road anomaly detection

Inputs: wname: $\mathrm{dB} 2$, the dual basis wavelet function; Level: 2, wavelet decomposition levels; dataset: $\mathrm{f}(\mathrm{x})$, sensed road surface conditions by an accelerometer (sample acceleration).

$1 \quad W(j, k)$ is Computed as the set of wavelet coefficients at each scale using (4).

2 At each scale, the power of the correlated sensed data $\mathrm{W}_{2}(j, k)$ is rescaled to the wavelet coefficient at that scale $W(j, k)$ using $(5)$

3 The proposed $A_{t h}$ is computed using (7)

4 The proposed $A_{\text {th }}$ is apply to threshold the correlated wavelet coefficients in step (3)

$5 \quad$ Find peaks in the threshold correlated wavelet coefficients

6 Determine signal boundaries and edges using the peaks in step 6

7 A spatial filter is generated using the edge location based on a unit step rectangular window function

8 Multiply the output signal from the spatial filter by the correlated wavelet coefficients in step 3 to obtain the denoised signal called "sigden"

9 Remove any transient fluctuation in the denoised signal "sigden" and saved as NSD

10 Compute the absolute value of NSD and save as variable NS

11 Create a counter to count and saves the number of spikes within a specified window in "NS" that corresponds to the number of road anomaly in the input dataset 
Similarly, results in Table 2 concerning road bump anomalies indicate that our proposed ATT and the ROHT algorithm achieved the same performance rate of $100 \%$ in the detection, precision and accuracy rates, respectively. This performance was followed closely by the manual threshold approach, which produced $67 \%$ road anomaly detection rate. The Otsu Multi threshold algorithm performed poorly with $33 \%$ detection rate. We observed similar performance rates when these algorithms were applied to other datasets. Thus, our findings suggest that our proposed ATT generally performed better than the Manual, Otsu Multithreshold and the ROHT algorithms compared in this work.

Table 1. Performance analysis of different threshold techniques applied to datasets having pothole anomalies

\begin{tabular}{|c|c|c|c|c|c|c|c|c|}
\hline & \multicolumn{5}{|c|}{ Performance Metrics } & \multirow{2}{*}{$\begin{array}{c}\text { Detection Output } \\
\text { Number of } \\
\text { Detected } \\
\text { Anomalies }\end{array}$} & \multicolumn{2}{|c|}{ Characterization Output } \\
\hline $\begin{array}{l}\text { Types of } \\
\text { Threshold }\end{array}$ & $\begin{array}{c}\text { Scale Space } \\
\text { Threshold }\end{array}$ & Accuracy & Precision & $\begin{array}{c}\text { True } \\
\text { Positive } \\
\text { Rate }\end{array}$ & $\begin{array}{c}\text { False } \\
\text { Positive } \\
\text { Rate }\end{array}$ & & $\begin{array}{c}\text { Number of } \\
\text { Bumps }\end{array}$ & $\begin{array}{c}\text { Number of } \\
\text { Potholes }\end{array}$ \\
\hline Manual & 0.0060 & 0.9963 & 0.6000 & 1.0000 & 0.0037 & 5 & 1 & 4 \\
\hline $\begin{array}{l}\text { Proposed } \\
\text { Adaptive }\end{array}$ & 0.0094 & 0.9981 & 0.7500 & 1.0000 & 0.0019 & 4 & 2 & 2 \\
\hline Multi-Thresh & 0.1316 & 0.9926 & 0.0000 & 0.0000 & 0.0019 & 1 & 1 & 0 \\
\hline ROHT & 0.0180 & 0.9926 & 0.3333 & 0.3333 & 0.0037 & 3 & 1 & 2 \\
\hline
\end{tabular}

Table 2. Performance analysis of different threshold techniques applied to datasets having road bump anomalies

\begin{tabular}{|c|c|c|c|c|c|c|c|c|}
\hline \multirow[b]{2}{*}{$\begin{array}{l}\text { Types of } \\
\text { Threshold }\end{array}$} & \multicolumn{5}{|c|}{ Performance Metrics } & \multirow{2}{*}{$\begin{array}{c}\text { Detection Output } \\
\text { Number of } \\
\text { Detected } \\
\text { Anomalies }\end{array}$} & \multicolumn{2}{|c|}{ Characterization Output } \\
\hline & $\begin{array}{c}\text { Scale Space } \\
\text { Threshold }\end{array}$ & Accuracy & Precision & $\begin{array}{c}\text { True } \\
\text { Positive } \\
\text { Rate }\end{array}$ & $\begin{array}{c}\text { False } \\
\text { Positive } \\
\text { Rate }\end{array}$ & & $\begin{array}{l}\text { Number } \\
\text { of Bumps }\end{array}$ & $\begin{array}{c}\text { Number of } \\
\text { Potholes }\end{array}$ \\
\hline Manual & 0.0100 & 0.9961 & 0.6667 & 0.6667 & 0.0019 & 3 & 2 & 1 \\
\hline Proposed & 0.0132 & 1.0000 & 1.0000 & 1.0000 & 0.0000 & 3 & 3 & 0 \\
\hline $\begin{array}{l}\text { Adaptive } \\
\text { Multi- } \\
\text { Thresh }\end{array}$ & 0.1409 & 0.9942 & 0.5000 & 0.3333 & 0.0019 & 2 & 1 & 1 \\
\hline ROHT & 0.0219 & 1.0000 & 1.0000 & 1.0000 & 0.0000 & 3 & 3 & 0 \\
\hline
\end{tabular}

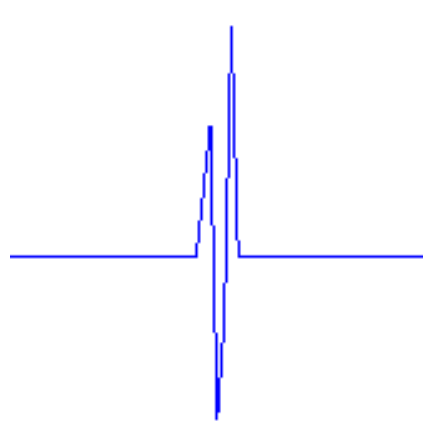

(a)

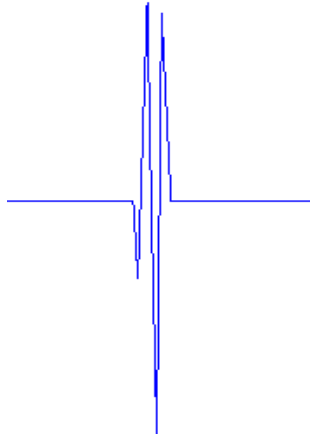

(b)

Figure 1. Output of different stages of RACA,

(a) Road Pothole Pattern (b) Road Bumps Pattern

\section{CONCLUSION}

In this paper, we have introduced a new adaptive threshold technique (ATT) combined with the Wavelet Transformation Scale Space Filtering (SSF) to develop an Adaptive WSSF (AWSSF) algorithm to improve road anomaly detection and characterization in vehicular technologies. Consequently, following our contribution, the AWSSF algorithm is able to adapt automatically and effectively to the random noise floor of an accelerometer's output measure acquired over asphalt road surfaces. Our findings suggest that using the proposed ATT in the AWSSF algorithm leads to higher accuracy, precision and lower false positive rates as compared to other adaptive methods compared with in this paper. Nevertheless, we note that the AWSSF can be further improved by optimizing the parameter values of the algorithm, an idea we shall consider in future works. 


\section{REFERENCES}

[1] F. Seraj, B. J. V. D. Zwaag, A. D. T. Luarasi and P. Havinga, "RoADS: A road pavement monitoring system for anomaly detection using smart phones," in In Proceedings of the 1st International Workshop on Machine Learning for Urban Sensor Data, (SenseML 2014), Netherlands, 2014.

[2] H. Bello-Salau, A. M. Aibinu, E. N. Onwuka, J. J. Dukiya, and A. J. Onumanyi, "Image processing techniques for automated road defect detection: A survey," in 11th International Conference on Electronics, Computer and Computation (ICECCO), 2014, 2014, pp. 1-4.

[3] J. M. Celaya-Padilla, C. E. Galván-Tejada, F. López-Monteagudo, O. Alonso-González, A. Moreno-Báez, A. Martínez-Torteya, J. I. Galván-Tejada, J. G. Arceo-Olague, H. Luna-García, and H. Gamboa-Rosales, "Speed bump detection using accelerometric features: a genetic algorithm approach," Sensors, vol. 18, pp. 443, 2018.

[4] H. Bello-Salau, A. M. Aibinu, E. N. Onwuka, J. J. Dukiya, A. J. Onumanyi, and A. O. Ighabon, "Development of a Laboratory Model for Automated Road Defect Detection," Journal of Telecommunication, Electronic and Computer Engineering (JTEC), vol. 8, pp. 97-101, 2016.

[5] L. C. González-Gurrola, F. Martínez-Reyes and M. R. Carlos-Loya, "The Citizen Road Watcher-Identifying Roadway Surface Disruptions Based on Accelerometer Patterns," in Ubiquitous Computing and Ambient Intelligence. Context-Awareness and Context-Driven Interaction, Ed: Springer, pp. 374-377, 2013.

[6] R. Madli, S. Hebbar, and P. Pattar, "Automatic Detection and Notification of Potholes and Humps on Roads to Aid Drivers," IEEE Sensors Journal, vol. 15, pp. 4313-4318, 2015.

[7] M. I. Strutu and D. Popescu, "Accelerometer Based Road Defects Identification System," U.P.B. Sci. Bull., Series C, vol. 76(3), 2014.

[8] A. Mukherjee and S. Majhi, "Characterisation of road bumps using smartphones," European Transport Research Review, vol. 8, pp. 1-12, 2016.

[9] N. Akhtar, K. Pandey and S. Gupta, "Mobile Application for Safe Driving," in Communication Systems and Network Technologies (CSNT), 2014 Fourth International Conference on, pp. 212-216, 2014.

[10] H. Bello-Salau, A. M. Aibinu, E. N. Onwuka, J. J. Dukiya, M. E. Bima, A. J. Onumanyi, and T. A. Folorunso, "A New Measure for Analyzing Accelerometer Data towards Efficient Road Defect Profiling Systems," Journal of Scientific Research and Reports, vol. 7, pp. 108-116, 2015.

[11] R. S. Figliola and D. Beasley, Theory and design for mechanical measurements: John Wiley \& Sons, 2015.

[12] H. Bello Salau, A. M. Aibinu, A. J. Onumanyi, E. N. Onwuka, J. J. Dukiya, and H. Ohize, "New Road Anomaly Detection and Characterization Algorithm for Autonomous Vehicles," Journal of Applied Computing and Informatics, 2018.

[13] Y. C. Tai, C. W. Chan and J. Y. J. Hsu, "Automatic road anomaly detection using smart mobile device," in conference on technologies and applications of artificial intelligence, Hsinchu, Taiwan, 2010.

[14] A. Mohamed, M. M. M. Fouad, E. Elhariri, N. El-Bendary, H. M. Zawbaa, M. Tahoun and A. E. Hassanien, "RoadMonitor: An intelligent road surface condition monitoring system," in Intelligent Systems' 2014, ed: Springer, pp. 377-387, 2015.

[15] M. Perttunen, O. Mazhelis, F. Cong, M. Kauppila, T. Leppänen, J. Kantola, J. Collin, S. Pirttikangas, J. Haverinen, and T. Ristaniemi, "Distributed road surface condition monitoring using mobile phones," in Ubiquitous Intelligence and Computing, ed: Springer, pp. 64-78, 2011.

[16] M. Strutu, G. Stamatescu, and D. Popescu, "A mobile sensor network-based road surface monitoring system," in 17th International Conference on System Theory, Control and Computing (ICSTCC), 2013, pp. 630-634, 2013.

[17] V. Astarita, M. V. Caruso, G. Danieli, D. C. Festa, V. P. Giofrè, T. Iuele, R. Vaiana, "A mobile application for road surface quality control: UNIquALroad," Procedia-Social and Behavioral Sciences, vol. 54, pp. 1135-1144, 2012.

[18] V. Astarita, D. C. Festa, D. W. E. Mongelli and A. Tassitani, "New methodology for the identification of road surface anomalies," in Service Operations and Logistics, and Informatics (SOLI), 2014 IEEE International Conference on, pp. 149-154, 2014.

[19] A. Vittorio, V. Rosolino, I. Teresa, C. M. Vittoria and P. G. Vincenzo, "Automated sensing system for monitoring of road surface quality by mobile devices," Procedia-Social and Behavioral Sciences, vol. 111, pp. 242-251, 2014.

[20] A. Mednis, "Implementation of participatory sensing approach in mobile vehicle-based sensor networks," Baltic J. Modern Computing, vol. 1(1-2), pp. 1-8, 2013.

[21] Z. Li, I. V. Kolmanovsky, U. V. Kalabić, E. M. Atkins, J. Lu, and D. P. Filev, "Optimal State Estimation for Systems Driven by Jump-Diffusion Process with Application to Road Anomaly Detection," IEEE Transactions on Control Systems Technology, vol. 25, pp. 1634-1643, 2017.

[22] O. C. Ugweje, "Selective noise filtration of image signals using wavelet transform," Measurement, vol. 36, pp. 279-287, 2004.

[23] H. Ohize, A. J. Onumanyi, M. Dlodlo and H. Bello-Salau, "An Adaptive Wavelet-Based Scale Space Filtering Algorithm for Spectrum Sensing in Cognitive Radio," in Wireless Communications and Networking Conference (WCNC), 2017 IEEE, 2017, pp. 1-6.

[24] D. Datla, A. M. Wyglinski, and G. J. Minden, "A spectrum surveying framework for dynamic spectrum access networks," IEEE Transactions on Vehicular Technology, vol. 58, pp. 4158-4168, 2009.

[25] N. Otsu, "A threshold selection method from gray-level histograms," Automatica, vol. 11, pp. 23-27, 1975.

[26] A. J. Onumanyi, E. N. Onwuka, A. M. Aibinu, O. C. Ugweje and M. J. E. Salami, "A modified Otsu's algorithm for improving the performance of the energy detector in cognitive radio," AEU-International Journal of Electronics and Communications, vol. 79, pp. 53-63, 2017. 